\title{
Cor triatriatum and total anomalous pulmonary venous connection to the coronary sinus
}

Kazutoshi Tachibana, MD, Nobuyuki Takagi, MD, Hisayoshi Osawa, MD, Motoki Takamuro, MD, Masato Yokozawa, MD, Hideki Tomita, MD, and Tetsuya Higami, MD, Sapporo, Japan

From the Department of Thoracic and Cardiovascular Surgery, Sapporo Medical University School of Medicine, Sapporo, Japan.

Received for publication Dec 30, 2006; accepted for publication Jan 8, 2007.

Address for reprints: Kazutoshi Tachibana, MD, Department of Thoracic and Cardiovascular surgery, Sapporo Medical University and hospital, S-1 W-16 chuo-ku, Sapporo, 060-8543 Japan (E-mail: aad72150@pop17.odn.ne.jp).

J Thorac Cardiovasc Surg 2007;134:1067-9

$0022-5223 / \$ 32.00$

Copyright $\odot 2007$ by The American Association for Thoracic Surgery doi:10.1016/j.jtcvs.2007.01.100

\section{Clinical Summary} PATIENT 1. A 22-day-

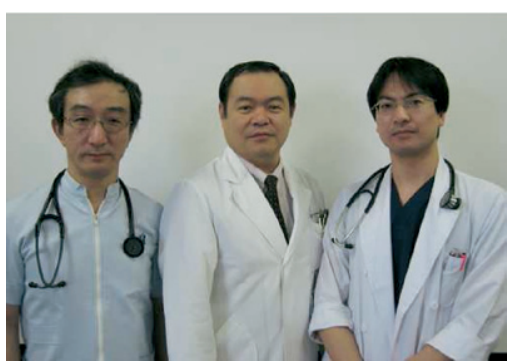

Drs Takagi, Higami, and Tachibana (left to right) old infant was referred to our hospital because of hypoxia. The neonate received ventilatory support. A twodimensional echocardiogram revealed the presence of coronary sinus (CS) total anomalous pulmonary venous connection (TAPVC) with a large atrial septal defect (ASD); the presence of a membrane within the left atrium (LA) was suspected. The neonate underwent an emergency operation with conventional cardiopulmonary bypass. The right atrium was opened, and drainage of all 4 pulmonary veins (PVs) into the CS was confirmed. There was no communication between the PVs and the LA. The membrane in the LA was identified through a secundum ASD. The

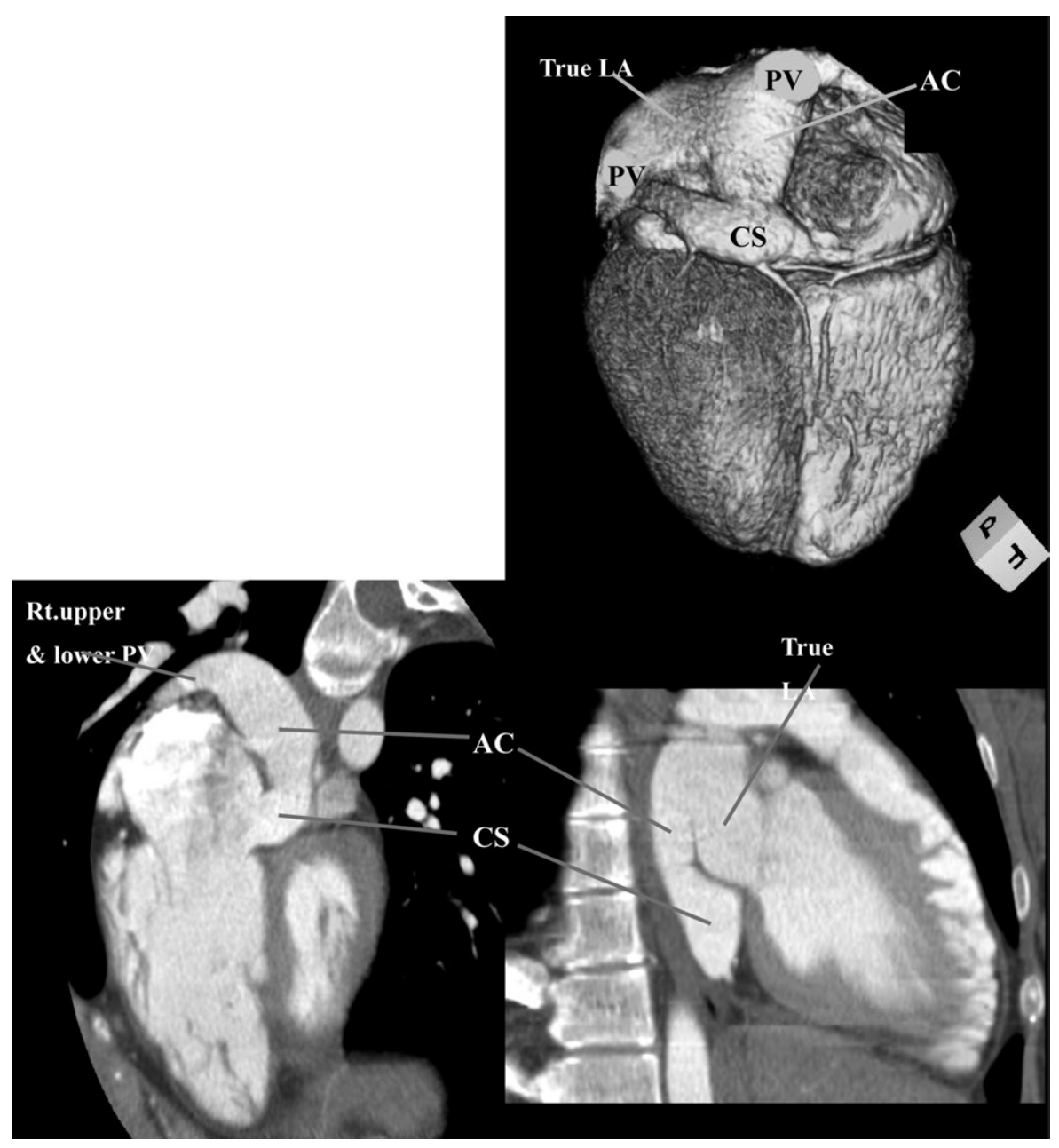

Figure 1. Three-dimensional computed tomographic scan (patient 2). The left atrium was subdivided into 2 chambers: the true left atrium (LA) and the accessory chamber (AC). The accessory chamber also communicates with the coronary sinus (CS) and receives the pulmonary veins (PVs). 
anomaly was easily repaired by means of complete excision of the membrane through the ASD. The CS was then "deroofed" and cut back into the LA. An autologous pericardial patch was sewn in place to reconstitute the atrial septum so that the CS (and thus the PVs) could drain into the LA. At follow-up 12 months after the operation, she remains well and is developing normally.

PATIENT 2. A 17-year-old girl was referred with the diagnosis of ASD. Echocardiography and cardiac catheterization raised the suspicion of an unroofed CS but could not confirm the diagnosis. Three-dimensional computed tomographic analysis provided really useful information and confirmed the presence of cor triatriatum with CS TAPVC (Figure 1). During cardiopulmonary bypass, the right atrium was incised longitudinally. The CS was noticeably enlarged. An ASD was not detected, and one was created in the position of the fossa ovale. There were communications between the dorsal chamber, which was receiving the PVs, and the ventral chamber, which had a left atrial appendage, and between the dorsal chamber and the CS. The obstructive membrane paralleled the posterior wall of the ventral chamber. The left- and right-sided components of the obstructive membrane were incised toward the orifice of the communication between the dorsal chamber and the CS (Figure $2, A)$. Then a tongue-like flap of the obstructive membrane was flipped down and anastomosed to the edge of the orifice (Figure $2, B)$. After completion of the intra-atrial rerouting with the obstructive membrane, a wide communication between the common PV and the LA was created, whereas the route of left-right shunting was closed, and the coronary vein was drained directly into the right atrium. After this operation, the patient was asymptomatic and returned to a normal school life.

\section{Discussion}

The embryogenesis of cor triatriatum remains controversial and is usually explained by citing the entrapment or malincorporation theory. ${ }^{1}$ The diverticulum, or common PV, grows toward the developing lungs and ultimately connects with the PVs, which have already formed. When this drainage pathway is established, the early connections between the splanchnic plexus and the umbilical-vitelline and cardinal venous system become of secondary importance and are largely lost. As the LA grows, the common PV is absorbed into its posterior wall, and the PVs enter the LA individually.

Depending on the stage at which normal embryogenesis is interrupted, a variety of connections of the AC to the atria and cardinal venous system can occur. If atresia of the common PV occurs very early, a major drainage route can be established through either the umbilicovitelline or cardinal venous system (total anomalous pulmonary venous connection). If stenosis occurs at a late stage, the result may be stenosis of the common PV (cor triatriatum). If stenosis occurs at a very late stage, after absorption of the common PV into the LA, the result will be stenosis or atresia of individual PVs. Concomitant cor triatriatum and total anomalous pulmonary venous connection, presented here, occur over the first 2 stages. In patient 2, normal embryogenesis might have been interrupted later than embryogenesis in patient 1.
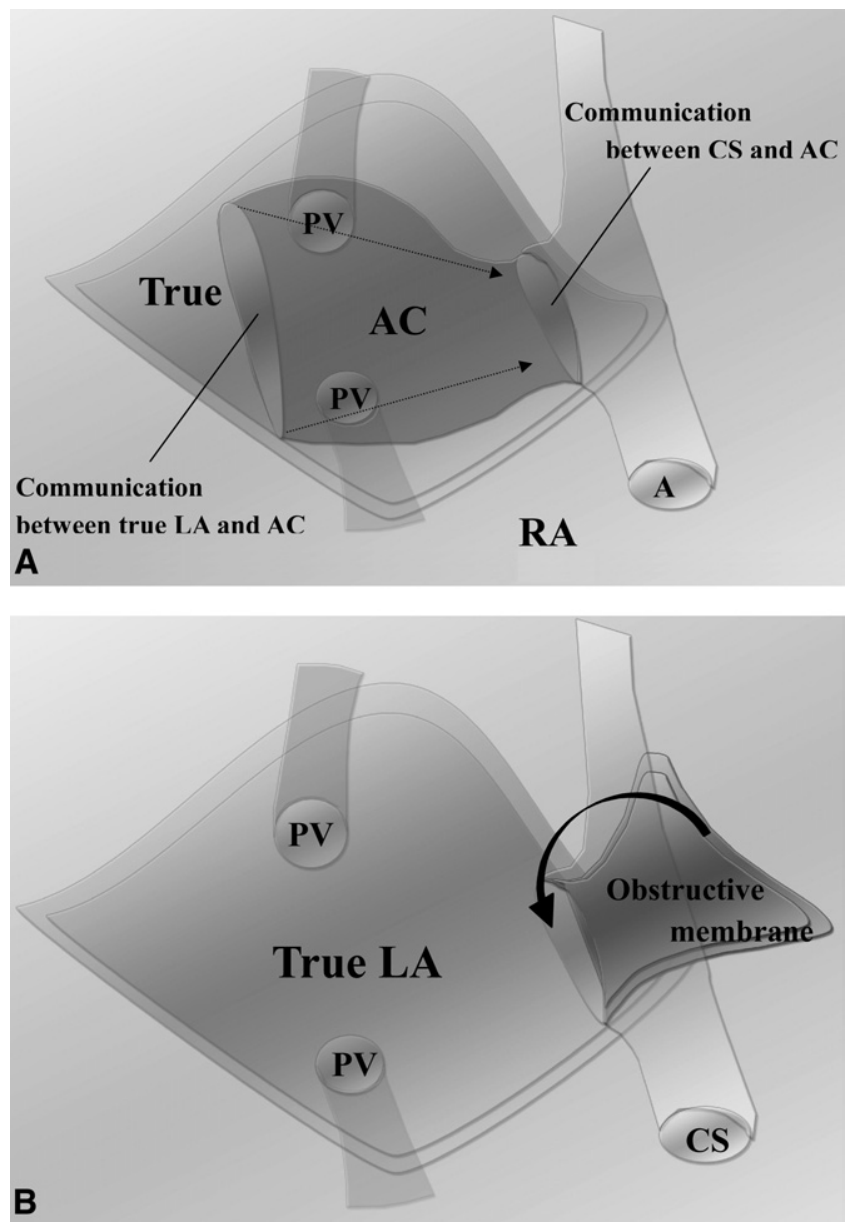

Figure 2. A, The left- and right-side obstructive membrane was incised toward the orifice of the communication between the accessory chamber $(A C)$ and the coronary sinus (CS). B, A tonguelike flap of the obstructive membrane was flipped down and anastomosed to the edge of the orifice. CS, coronary sinus; $L A$, left atrium; $R A$, right atrium; $P V$, pulmonary vein.

Careful two-dimensional echocardiography could be the procedure of choice in identifying the subdividing membrane. Unfortunately, cineangiography is often unsuccessful in demonstrating the abnormal left atrial membrane. However, a threedimensional computed tomographic scan provides really useful information for the evaluation of such a complicated association.

Both surgical repairs were achieved simply; notably, in patient 2 intra-atrial rerouting was completed with the obstructive membrane. Cor triatriatum has a lot of variations and can be repaired by using various methods. ${ }^{2,3}$ It is important to consider the possible use of the obstructive membrane and to repair as simply as possible.

In summary, although rare, concomitant cor triatriatum and TAPVC can be treated successfully if the diagnosis is confirmed in detail and sufficient information is available preoperatively. 


\section{References}

1. Van Praagh R, Corsini I. Cor triatriatum: pathological anatomy and a consideration of morphogenesis based on 13 post-mortem cases and a study of normal development of the pulmonary vein and atrial septum in 83 human embryos. Am Heart J. 1969;78:379-405.
2. Vouhe PR, Baillot-Venant F, Fermont L, et al. Cor triatriatum and total anomalous pulmonary venous connection: a rare surgically correctable anomaly. J Thorac Cardiovasc Surg. 1985;90:443-5.

3. Salomone G, Tiraboschi R, Bianchi T, Ferri F, Crippa M, Parenzan L. Cor triatriatum. Clinical presentation and operative results. J Thorac Cardiovasc Surg. 1991;101:1088-92.

\title{
Fatal intraoperative thrombosis in contemporary adult thoracic aortic surgery requiring deep hypothermic circulatory arrest: Observations from the literature, 1993-2006
}

\author{
John G. T. Augoustides, MD, FASE, Philadelphia, Pa
}

$\mathrm{F}$ atal intravascular coagulation in the setting of thoracic aortic surgery with deep hypothermic circulatory arrest (DHCA) was highlighted in a case series $(n=20)$ that identified exposure to aprotinin as a significant risk factor. ${ }^{1}$ The findings in this case series have since been attributed to inadequate heparinization because it was only appreciated thereafter that aprotinin prolongs the celite-based activated clotting time (ACT) ${ }^{2}$ However, even in the presence of adequate heparinization by kaolin-based ACT or heparin concentration, case reports of fatal thrombosis in thoracic aortic surgery with DHCA have persisted $(\mathrm{n}=5,1998-2006) .^{2-5}$ The purpose of this brief communication is to review this case series as a platform from which further research can be planned. The literature search was conducted with PubMed (last entry: May 21, 2007) with the following search terms: thrombosis and DHCA; aprotinin and thrombosis in DHCA; aminocaproic acid and thrombosis in DHCA; and tranexamic acid and thrombosis in DHCA.

As a group, the published case reports of adult DHCA thrombosis can be summarized as follows ${ }^{2-5}$ :

1. The 5 patients were all older than 60 years ( 2 men and 3 women).

2. All underwent thoracic aortic procedures with DHCA.

3. Factor V Leiden was identified in 2 of the 5 patients. ${ }^{4,5}$

4. All patients had antifibrinolytic therapy (3 with aprotinin and 2 with aminocaproic acid).

5. Anticoagulation was titrated with heparin to maintain the $\mathrm{ACT}$ at longer than 500 seconds, the heparin concentration

From the Cardiothoracic Section, Anesthesiology and Critical Care, Philadelphia, Pa.

Received for publication May 22, 2007; accepted for publication May 30, 2007.

Address for reprints: John G. T. Augoustides, MD, FASE, Cardiothoracic Section, Anesthesiology and Critical Care, Dulles 680, HUP, 3400 Spruce St, Philadelphia, PA 19104-4283 (E-mail: yiandoc@ hotmail.com).

J Thorac Cardiovasc Surg 2007;134:1069-70

$0022-5223 / \$ 32.00$

Copyright $\odot 2007$ by The American Association for Thoracic Surgery doi:10.1016/j.jtcvs.2007.05.033 at greater than $2.7 \mathrm{U} / \mathrm{mL}$, or both. The kaolin-based ACT was always used in the presence of aprotinin.

6. Circulatory collapse caused by intravascular thrombosis began in all cases within 60 minutes of separation from cardiopulmonary bypass and protamine administration.

7. The circulatory collapse was life-threatening and resistant to pharmacologic support. In all cases it necessitated return to cardiopulmonary bypass.

8. The degree of thrombosis was massive, involving the heart chambers, thoracic aorta, pulmonary arteries, and great veins. In 4 of 5 patients, the disseminated thrombus was evident intraoperatively. In the fifth patient autopsy revealed extensive thrombosis within the small pulmonary arteries that was responsible for fatal intraoperative right ventricular failure.

9. The syndrome caused intraoperative death in all 5 patients.

Of course, this small case series has the usual inherent limitations. There are many more questions than answers. However, the following observations might serve to direct further investigation of this lethal syndrome to improve its management:

1. A worldwide case registry would allow a greater case number for further in-depth analysis.

2. Hypercoagulable states, such as Factor V Leiden, might have a role in the pathophysiology of this syndrome.

3. In the presence of an antifibrinolytic, standard-of-care anticoagulation with heparin for adult DHCA does not protect all patients against serious intraoperative thrombotic complications.

4. Aprotinin is not the only antifibrinolytic associated with this syndrome. To date, no cases of this syndrome have been reported in association with tranexamic acid.

5. When a case occurs, the following considerations might improve clinical care:

a. Blood samples from the patient should be analyzed in consultation with a hematologist to identify possible mechanisms.

b. The index patient and family should be screened for underlying hypercoagulable states.

c. Patient autopsy might be required to determine the mechanism of death, especially if the thrombosis is not evident intraoperatively. 